\title{
Design of a Video Data Transmission System Based on Wireless Mesh Network
}

\author{
Liu-Qing YANG ${ }^{1, a}$ and Guo-An ZHANG ${ }^{2, b, *}$ \\ ${ }^{1}$ Information Technology Engineering Dept. of Fuzhou Polytechnic, Fuzhou, Fujian, China \\ ${ }^{2}$ Information Science and Engineering School of FJUT, Fuzhou, Fujian, China \\ a2220310780@qq.com, bzhang.guoan@fjut.edu.cn \\ ${ }^{*}$ Corresponding author
}

\begin{abstract}
Keywords: Video monitoring, Frequency planning, Data sampling, Mesh
Abstract. A video data sampling and transmission system is designed for video monitoring based on wireless mesh network. Discussion is made on the use of standards, working frequency, network infrastructure, coverage solutions, link budget, frequency planning, power supply, supporting transmission and AC design. Overall solution on wireless Mesh network digital video system is proposed and it is applied to practical wireless network video monitoring system, and nice results are achieved through practical application.
\end{abstract}

\section{Introduction}

Since safety requirements become more and more important, digital video surveillance networks have been widely used in various industries. The use of wireless Mesh network technology can quickly build video surveillance network to meet the requirements of economic and social development. In a mountainous area or terrain, scattered population living requires good coverage, but cable distribution network result in high cost. So IT is urgently needed for urban fringe or rural areas, especially the development of wireless access coverage.

\section{Design and Construction of a Wireless Data Sampling and Transmission System}

\subsection{Design Standards and Principles}

\section{1) Using Standard}

\section{(1) WiFi/WAPI:}

Thin AP devices which support WiFi/WAPI standard are used for networking.

(2) 802.11n:

Devices which meet the $802.11 \mathrm{n}$ standard are mainly used for networking. $802.11 \mathrm{n}$ selecting factors include hot spots type, design capacity, network development[1]. For hot spots in high demand of network capacity, $802.11 \mathrm{n}$ devices should be used directly for construction.

\section{2) Working Frequency and Principles of Application}

(1) Working frequency [2]:

WLAN $802.11 \mathrm{n}$ work in the $2.4 \mathrm{GHz}$ band, the frequency ranges from 2.400 to $2.4835 \mathrm{GHz}$. Total bandwidth of $83.5 \mathrm{M}$ is divided into 13 subchannels, bandwidth of each subchannel $22 \mathrm{MHz}$; among which three channels have no interference. Generally, the proposed frequency points are 1,6,11.

For WLAN $802.11 \mathrm{n}$ devices working in $5.8 \mathrm{GHz}$ band, the frequency ranges from $5.725 \mathrm{GHz}$ to $5.850 \mathrm{GHz}$, a total bandwidth of $125 \mathrm{MHz}$ is divided into five channels, bandwidth of each channel is 20MHz. These non-overlapping channels can all be used.

(2) The principle of frequency applications

2.4GHz band selection: There are many interferences in $2.4 \mathrm{GHz}$ band, such as interferences from other network operators, owners self-built network. 20M bandwidth should be adopted for networking and 
planning, three non-overlapping channels $1,6,11$ should be selected. If independent operators are allowed to distribute network, with less interference and fewer number of AP, $40 \mathrm{MHZ}$ or a $20 \mathrm{M}$ with a 40M-band can be used for networking.

$5.8 \mathrm{GHz}$ band selection: there is relatively less interference in $5.8 \mathrm{GHz}$ band, so two $40 \mathrm{M}$ with one $20 \mathrm{M}$ should be adopted for networking (corresponding to three frequency spots with the $2.4 \mathrm{GHz}$ ), or five 20M-band networking (for intensive case of AP deployment). In the area with less interference or only independent distributing network operators are allowed, one or two $40 \mathrm{M}$ networking solutions can be used. Indoor distribution system does not support the $802.11 \mathrm{n}$ devices under 5.8G band, devices under $2.4 \mathrm{G}$ should be used. The case of independent construction, if possible, the $5.8 \mathrm{G}$ band should be applied to reduce interference.

\section{3) AP Emission Power}

According to specifications of China's telecommunications industry wireless LAN AP, AC equipment, AP emission power should meet following requirements:

(1) For mounted indoor $802.11 \mathrm{n}$ thin AP (with two spatial streams, supports dual-band $2.4 \mathrm{G}$ and $5.8 \mathrm{G}$ simultaneously), RF output equivalent isotropic radiated power $\leq 20 \mathrm{dBm}$;

(2) Indoor division and combined type $802.11 \mathrm{n}$ thin AP (single spatial stream, supports $2.4 \mathrm{GHz}$ band), AP device output power $\leq 27 \mathrm{dBm}$, and RF output equivalent isotropic radiated power $\leq 20 \mathrm{dBm}$;

(3) Outdoor $802.11 \mathrm{n}$ thin AP, adjustable emission power, RF output equivalent isotropic radiated power $\leq 27 \mathrm{dBm}$;

(4) When combined construction methods are used, antenna port power should comply with national requirements for electromagnetic radiation protection.

\subsection{Design of Mesh Transmission System}

\section{1) Network Architecture}

Various types of AP are included in wireless access points. In wireless access points, thin AP devices should be applied to simplify installation and upgrade, and to meet the requirements of centralized management for a network. Cable connection is commonly used between AP and switch/ONU, with no more than 100 meters transmission distance, usually no more than 80 meters. When the transmission distance go beyond the length of cable transmission, connection such as optical switches can be used. Each AP can be converged by switch/ONU. According to the number of hot spots and distribution, 1-2 levels of switch/ONU can be used. With large quantities and wide distribution of AP, an aggregation switch should be used to converge each access switches. With small amount of AP and concentrating distribution, one level of switch/ONU is enough. In hotspots with PON access conditions, part or all of the switches can be replaced by ONU. With higher level of security needs, the hotspot aggregation switches should get one backup (as the aggregation switch 2 in Figure 3), for redundant aggregation nodes or in the uplink and downlink, to improve the reliability of the network. If there is no conditions for a wired connection between switch and $\mathrm{AP} / \mathrm{ONU}$, a wireless $5.8 \mathrm{GHz}$ bridge can be used. Wireless bridges should follow some principles: $2.4 \mathrm{GHz}+5.8 \mathrm{GHz}$ dual-band AP should be used, where $2.4 \mathrm{GHz}$ frequency covers user, $5.8 \mathrm{GHz}$ frequency as wireless backhaul.

\section{2) Coverage Solutions}

(1) AP independent installed

a)AP directly covers indoor target region through a pair of antenna;

b)Frequency interval and environment should be noted in the search of AP placement points;

c) The antenna should be installed at the location where target region can well be covered. If special needs to beautify the environment are considered, an external antenna should be installed on a concealed position with little impact on covering effect. or the use of landscaping antenna; the antenna must be placed outside of metal ceilings;

d)Antenna can either be mounted directly on the antenna port of AP, or be connected via coaxial cable. To avoid unnecessary loss, the distance between AP and its antenna should be shortened. 
(2) AP combiner indoor distributed antenna system

a)By the use of building, the existing or new distribution system should be moved to combine WLAN signal;

b)Indoor distributing system components are required to support $2.4-2.5 \mathrm{GHz}$ frequency band;

c)Distance between indoor division system antennas must meet the requirements of WLAN coverage;

d)According to AP output power, under the premises to meet coverage and interference isolation requirements, number of combined nodes should be reduced;

e)Distribution system can be combined with AP direct coverage to cover the target area. AP direct coverage is used to solve capacity.

(3) AP access to a small distribution system

a) On hot spot with no indoor distribution system, where target coverage area is a plurality of continuous separating space, and the traffic is not big, an AP is connected to multiple antennas to cover the target area, in order to achieve a small distributing system in local area;

b) To select the power of AP according to the actual need, power is allocated to each antenna by power splitter / coupler, output power on each antenna port ranges from 10 to $15 \mathrm{dBm}$, shall not exceed $20 \mathrm{dBm}$.

(4) AP outdoor coverage

a)Outdoor AP is used to cover indoor target area;

b) Taking into account penetration loss of building, when the thickness of the building does not meet the requirements of loss, several AP should be used to cover from multiple directions;

c) Distance from antenna to target buildings should be within $100 \mathrm{~m}$;

d)According to height to hang antenna and horizontal / vertical lobe angle, ability of an antenna horizontal and vertical coverage can be estimated, width and height of building should be within the coverage range of the antenna, and declination of the antenna should be noted.

(5) Mesh networking

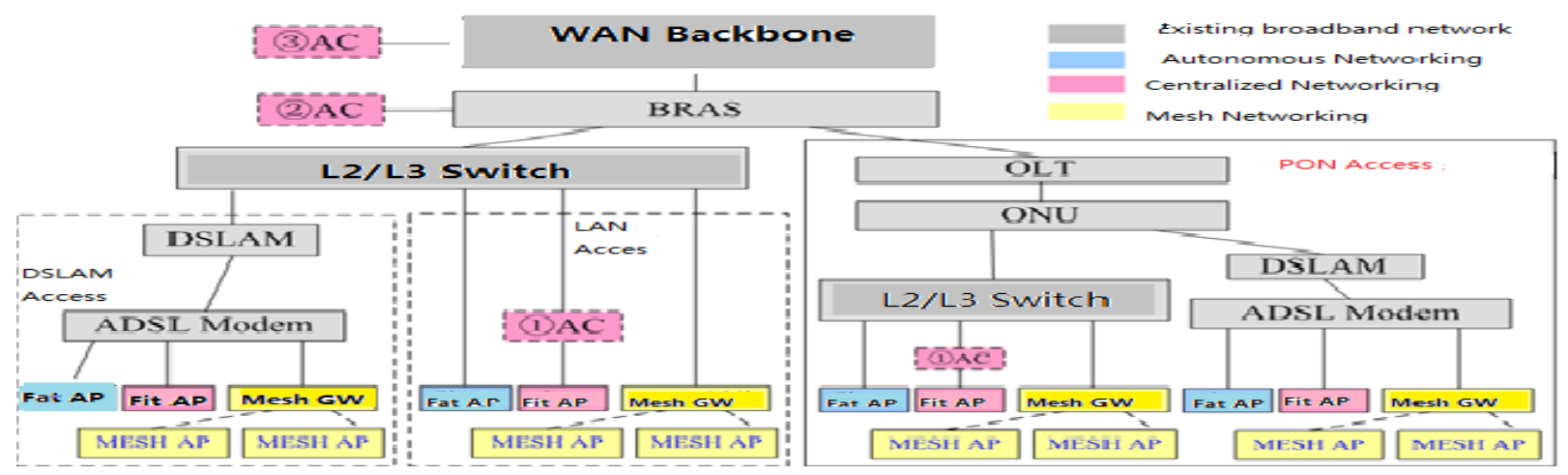

Fig. 1. WLAN network architecture based on Mesh

Mesh networking is one kind of network architecture based on multi-hop routing, where Root AP and Mesh AP are jointly used to cover a contiguous area.Network architecture are shown in Figure 1.Among them:

a) Root AP: refers to AP connected to the upper level network via a wired way. Root AP enables users to access and AP aggregation. In placement of Root AP, transport and electricity are needed to provide;

b) Mesh AP: This refers to AP backhauled signal to the Root AP by wireless way, where local user access is achieved. In placement of Mesh AP, only electricity is required.

\section{1) Link Budget}

(1) Outdoor propagation model 
Free-space propagation model can be used on open space, the propagation loss of electromagnetic waves in a free space path is consistent with:

$$
L_{0}(d B)=92.4+20 \lg (d)+20 \lg (f)
$$

Where: $\mathrm{L}_{0}$ : free space loss (unit: $\mathrm{dB}$ ); $\mathrm{D}$ : transmission distance (unit: $\mathrm{Km}$ ); $\mathrm{F}$ : working frequency (unit: $\mathrm{GHz}$ ). Respectively taking: $\mathrm{d}=0.1$ to $0.5 \mathrm{Km}, 1$ to $5 \mathrm{Km} ; \mathrm{f}=5.8 \mathrm{GHz}$, and $\mathrm{d}=0.01$ to $0.05 \mathrm{Km}, 0.1$ to $0.5 \mathrm{Km}, \mathrm{f}=2.45 \mathrm{GHz}$; calculating results are shown in Figure 2.

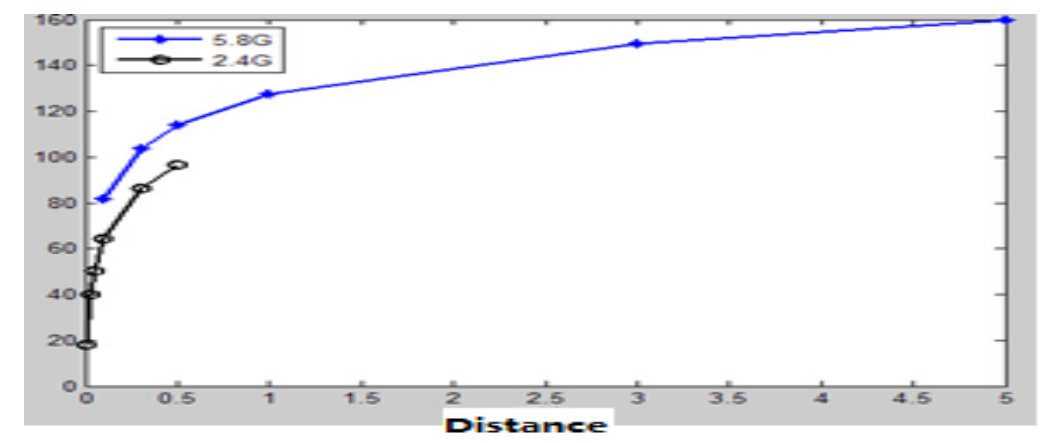

Fig. 2. Propagation loss of electromagnetic waves in free space path

(2) Indoor attenuation factor [3]

In indoor environment, an attenuation factor model can be selected for indoor radio propagation model, its expression is:

$$
P L(d)[d B]=P L\left(d_{0}\right)+10 n \lg \left(\frac{d}{d_{0}}\right)+\mathrm{S} F
$$

Wherein: PL $(\mathrm{d} 0)=20 \lg (4 \Pi \mathrm{d} 0 / \lambda)$, and generally take $\mathrm{d} 0=1 \mathrm{~m}$, when the frequency is $2.45 \mathrm{GHz}$, its value is $40 \mathrm{~dB}$; $\mathrm{n}$ : attenuation factor, is associated with the propagation environment, can be seen in Table 1; SF: additional attenuation factor, refers to the additional loss caused by floor, partitions, walls, etc.

Table 1. Typical attenuation factor

\begin{tabular}{cc}
\hline Environment & $\mathrm{n}$ \\
\hline Full open environment & $2.0 \sim 2.5$ \\
Semi-open environment & $2.5 \sim 3.0$ \\
Relatively closed environment & $3.0 \sim 3.5$ \\
\hline
\end{tabular}

Theoretical calculation of coverage effect: If the maximum transmission distance in engineering design ranges from 20 to $60 \mathrm{~m}, \mathrm{n}$ is from 2 to 3.5, results are shown in Figure 3.

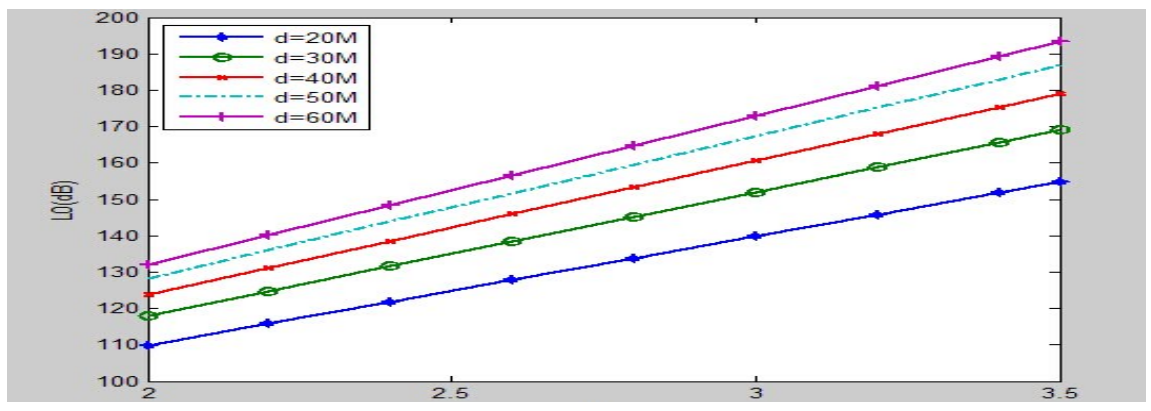

Fig. 3. Indoor radio propagation model under attenuation factor $n$ 
WLAN frequency planning should follow the following principles:

(1) The location of a signal source will have a great impact on the effect of signal coverage and interference, so it should be reasonably placed to make it conducive to signal coverage and minimal interference with each other based on the actual building, partitions circumstances.

On outdoor and indoor overall open area, cellular layout should be used, including a uniform distribution of the signal in horizontal and vertical directions, the frequency multiplexing for WLAN.

(2) The coverage area of each AP Shall be shown according to the distribution of users within the coverage area and design diagram, then possible blind spot region may be analyzed. Considering interference between AP, especially interference with existing AP, laying of AP should be adjusted. In addition, mutual interference of adjacent floors AP will appear, so pay special attention to consideration of the coverage area of each AP from a three-dimensional view.

(3) Overlap area between AP coverage regions can ensure seamless coverage and adapt to load balancing; distance between different AP in the same channel should be controlled to reduce interference.

(4) When AP and other mobile communication networks (such as TD-SCDMA, TD-LTE) are built on the same site, isolation between different systems should meet technical requirements.

(5) Mounting location of AP antenna should be far away from electronic equipment, or to shield the source of interference such as microwave ovens, monitors, motors, etc. if necessary.

\section{3) Device Power Supply [5]}

AP power can be POE (Power over Ethernet) power supply, AC power supply, DC power supply; various power supply should be used with the following principles:

(1) POE power supply must not exceed 100 meters away, usual distance should be controlled within 80 meters. There are three ways in POE power supply: POE power supply module, POE switch, ONU2 supported by POE.

a) In situation of POE switch or ONU supporting POE, equipment supply is prefered;

b) When using POE mode power supply switch or ONU supporting POE, the total output power should be assured to meet the total power requirements of the multiple connected AP;

c) When using POE power supply switch, or ONU supporting POE, POE switch requires a good grounding;

If switch or ONU does not support POE, or there is insufficient power supply capacity of related equipment, POE power supply module can be used to supply power to the AP.

(2) For some higher power consumption of the device (including 802.11n standard AP, smart antennas AP, etc.) requires the use of POE + switches, dedicated POE power supply module, AC or DC power supply. While no special circumstances is described, referred POE include both cases of POE and POE+switch modes. POE switch and ONU which supports POE refer to Ethernet switch or ONU with built-in POE power supply modules. Important hotspots and hotspot where power supply can not be guaranteed electricity supply of AP should be equipped with a backup power supply.

\section{1) Supporting the Transmission Part}

Several ways such as PON, PTN, SDH access are adopted in the construction of supporting transmission part in WLAN hotspot. According to differences in local business needs, the transmission bandwidth requirements can be calculated with reference to the following methods:

Transmission bandwidth $=$ number of concurrent users $\times$ single-user speed;

Transmission bandwidth $=$ number of AP $\times$ average transfer speed of a single AP.

In addition, the transmission bandwidth can also be calculated through other appropriate methods. On the basis of above bandwidth calculation results, a certain margin should be set aside.

\section{2) AC (Access controller) design}

(1) AC design position:

To meet the needs of AP access and network management, AC device should be designed based on the MAN construction of region. In area with MAN built, logic position of $\mathrm{AC}$ devices is equivalent 
with access control layer of MAN, and its physical location should be centralized to deploy for centralized management of AP. When AC is forwarding data, centralized forwarding mode should be used, i.e., the user data stream from AP aggregates into AC via the data tunnel between the AP and $\mathrm{AC}$, then is uniformly forwarded by AC. In particular scenarios of colleges and universities, if the user data stream from AP does not pass through the company's public network (such as traffic in a campus network, or traffic in a internal network of group customers), this part of the data stream can be forwarded through local network. For AC deployment location, it should be based on situation of the regional network construction, the business development needs, equipment performance. AC can hang aside or be serially connected to core router, aggregation switches or BRAS (Broadband Remote Access Server). To avoid data roundabout generated by hanging beside, the new network is preferred to build in series connection.

(2) AC design capacity:

It is recommended that the number of AP managed by AC should not exceed $70 \%$ of AC capacity.

(3) AC backup policies:

To improve network reliability, another AC should be used for backup.

\section{Overall Design of Wireless Network Video Monitoring System}

\subsection{System Architecture}

A wireless network video monitoring system consists of monitoring front-end, wireless network link, the monitoring center / monitoring client. When users do not need to constantly record, the monitoring center functions can be canceled, then the system only consist of the monitoring front-end and monitoring client. As long as the front-end equipment is working, video surveillance can be done in any wireless network coverage location, not constrained by the center and the relative area.As a result, digitized video transmission system is realized in the true sense.

\subsection{Monitor Front End}

Monitor front end consists of wireless network devices and monitoring camera, output of the monitoring camera is a fully digital conversion, the interface of which is simple and easy to link. The camera can be a gun style machine, or a ball style machine. After the camera video is captured by wireless device, it is sent to the monitoring center to establish monitoring video streams. For traditional analog cameras, network video server can access the camera's video interface. With a combination of network video server and a wireless network, the front-end video is transmitted to the monitoring center via a wireless link [7].

\subsection{Network Link [8]}

A wireless video professional network can be made by a single or multiple wireless network devices based on system requirements, where the wireless devices adopt standard $802.11 \mathrm{n}$ protocols, and $5.8 \mathrm{GHz} / 2.4 \mathrm{GHz}$ band is used for data transmission. A wireless video network can be a structure composed of point to multipoint, multilevel relay, point to point network. For safety performance in the transmission channel, MAC address filtering and 128-bit WEP/WPA/WPA2/dual-layer isolation are used, end-user's connection with a wireless device is limited to protect the safety of video channels[9]. Multipolarized antenna design, antenna power, and wireless network management make it possible for video communication and flexible networking over more than 10 kilometers through software control.

\subsection{Monitoring Center and Client [10]}

A monitoring center consists of one server, one monitoring PC or large-screen monitor. Software system of the server completes video forwarding from front-end video surveillance point, and video server recording. Client software on monitoring PC can connect to the server software system 
according to demand, get video streams from server-side forwarding. Video information is also available directly from the front-end control point. Clients can implement video monitoring, control, alarm, record and historical retrieval.

\section{Conclusion}

During overall system design, hierarchical and systematic network structure is used, and function model of different layers are strictly defined, attention to different characteristics of the configuration are paid on different layers. By reasonable analysis and practical tests, AP's actual coverage and capacity are obtained. Has been applied in software industrial campus, fishing port, highway construction projects and other security monitoring, this system achieved reasonably economic and social benefits. However, maintenance services of a wireless Mesh network monitoring systems is more complex than that of wired communication systems. First of all, a wireless Mesh network is operating in unauthorized frequency band, which has got more serious interference compared with other wireless communication systems. Secondly, AP in a wireless Mesh network, indoor distribution systems are typically deployed under the public environment without reliable power supply and physical protection. All of these factors have brought greater uncertainty to the running quality of a wireless Mesh network, increased the difficulties of wireless Mesh network maintenance. Nevertheless, there is still an important reference value in the construction of a video surveillance system (e.g.: video surveillance on campus and dangerous positions).

\section{Acknowledgments}

Project supported by Science \& Technology Foundation of Fuzhou, China(Grant No.2013-G-96, No.2017-G-93 ) .

\section{References}

[1] Eldad Perahia , Robert Stacey, "Next Generation WLAN,"[M]. Beijing: People's Posts and Telecommunications Press, 2010.9:9-13.

[2] Xie Xi-re,Computer Network(6th ed.)[M].Beijing:Electronic Industry Press,2013.6:360-378.

[3] Zhanji $\mathrm{Wu}$ and Xiang Gao,Improved MIMO-OFDM seheme for the next generation WLAN,[J].Journal of Systems Engineering and Electronics,2012.24(1):.52-59.

[4] Hu Zhi-yuan,Wang Xiao-xiang and Zhang Xian, "Reliable multicast in wireless mesh networks based on routing quality,"[J].Journal on Communications, 2011.32(5):1-7.

[5] Jianjun Li, "Design and Implementation of IaaS based architecture cultural wisdom"[D]. Soochou University Master Thesis, 2014.11,pp20-21.

[6] Liang Du-guo,Zhang Yan-xia and Cao Ning "Network video monitoring technology and intelligent application," [M].Beijing: People's Posts and Telecom Press,2013:233-260.

[7] Cao Fang, "Wireless video surveillance, security battlefield force,"[J]. Shanghai Informatization, 2013(8):35-39.

[8] Li Fang-fang, Wang Jing and Chen Cun-xiang, Method of performance evaluation for wireless mesh network. [J].Application Research of Computers, 2013.30(2): 551-553.

[9] OE Muogilim, KK Loo and R Comley,"Wireless mesh network security: A traffic engineering management approach", Journal of Electrical and Computer Engineering, 2011, 34(2):478-491.

[10]Xi Sha-zi, Detailed of intelligent network video surveillance technology and practice.[M]. Beijing: Tsinghua University Press,2010.348-361. 